\title{
Preparedness for Pediatric COVID-19 Using Systems Approach: Experience of a Health Care Facility from India
}

\author{
Shivakumar Hiremath ${ }^{3}$ \\ ${ }^{1}$ Department of Pediatrics, K.S. Hegde Medical Academy, Nitte \\ (deemed to be University), Mangaluru, Karnataka, India \\ ${ }^{2}$ Department of Community Medicine, K.S. Hegde Medical \\ Academy, Nitte (deemed to be University), Mangaluru, Karnataka, \\ India \\ ${ }^{3}$ Department of Hospital Management, K.S. Hegde Medical \\ Academy, Nitte (deemed to be University), Mangaluru, Karnataka, \\ India
}

Rathika D. Shenoy ${ }^{1, \odot ~ P r a v e e n ~ N a y a k ~}{ }^{1} \quad$ Ankeeta Menona Jacob² Swathi Sunil Rao ${ }^{1}$

\begin{abstract}
Address for correspondence Rathika Damodara Shenoy, PhD, Department of Pediatrics, K.S. Hegde Medical Academy, Nitte (deemed to be University), Nithyananda Nagar, Deralakatte, Mangaluru, Karnataka 575 018, India

(e-mail: rathika.shenoy@nitte.edu.in).
\end{abstract}

\begin{abstract}
Keywords

- children

- health care system

- infrastructure

- modifiable factors

- pandemic

- systems approach

A pandemic leads to disruption and stretching of an existing health care system and its resources. Coronavirus disease 2019 (COVID-19) data show distinct and severe manifestations in children necessitating critical care. Children need prioritization as they are susceptible to COVID-19 as part of the family cluster, varied presentations, and mortality. The purpose of this report is to discuss the optimization of the health care system for pediatric care. The key initiatives were to identify our objectives, bring out changes to the organizational processes, and integrate the same into the existing system. A systems approach to health care delivery by optimizing infrastructure, human resources, materials, funding, leadership, and governance was undertaken. This resulted in creation of distinct COVID-19 and non-COVID-19 service areas, management protocols, and trained health care workers. Partnership was forged with the public health system. This preparedness and continued responses to the demands of the health care system helped us manage children ranging from neonates to adolescents efficiently. Though the health care system is mostly open, acting upon the modifiable factors gave better preparedness in a short time.
\end{abstract}

\section{Introduction}

The coronavirus disease 2019 (COVID-19), caused by severe acute respiratory syndrome coronavirus 2 , is thought to largely spare the children. Data from China and the United States suggest that pediatric COVID-19 constitutes just $~ 2.0 \%$ of all reported positive..$^{1-4}$ Children of all ages are susceptible to COVID-19, including newborns. ${ }^{5}$ Among them, those with respiratory failure due to severe acute respiratory infection
(SARI) or multiorgan dysfunction syndrome due to multisystem inflammatory syndrome in children (MIS-C) require critical care. ${ }^{3,46-9}$ In a scenario of $5 \%$ cumulative pediatric infection proportion, the projected estimate of sick children requiring hospitalization is just $\sim 0.2 \%{ }^{10}$ World over, several pediatric intensive care units (PICU) are converted to medical ICUs due to the disproportionately severe illness and progression in adults. ${ }^{3,11,12}$ published online

July 6, 2021
DOI https://doi.org/

$10.1055 / \mathrm{s}-0041-1731605$

ISSN 2582-4287 (c) 2021. Nitte (Deemed to be University).

Nitte University. This is an open access article published by Thieme under the terms of the Creative Commons Attribution-NonDerivative-NonCommercial-License, permitting copying and reproduction so long as the original work is given appropriate credit. Contents may not be used for commercial purposes, or adapted, remixed, transformed or built upon. (https://creativecommons.org/licenses/by-nc-nd/4.0/). Thieme Medical and Scientific Publishers Pvt. Ltd. A-12, 2nd Floor, Sector 2, Noida-201301 UP, India 
The COVID-19 pandemic supposedly started in late November 2019 from the Chinese city of Wuhan. India reported its first case on January 30, 2020 in a student returning from Wuhan. The World Health Organization (WHO) declared COVID-19 as a pandemic on March 11, 2020, and around this time, India accounted for just $\sim 350$ cases.$^{13}$ China, Korea, and the United States reported their first pediatric case on January 20, February 19, and March 2, respectively. ${ }^{3}$ On April 26, 2020, the National Health Service of the United Kingdom gave the first alert on children presenting critically ill with overlapping features of toxic shock syndrome and Kawasaki disease, later described to be temporally associated with COVID-19. On May 14, the Centers for Disease Control and Prevention, United States, released the MIS-C advisory.

A pandemic leads to disruption and stretching of an existing health care system and its resources to its limit. Pediatric cases span from neonates to adolescents with different requirements. We discuss the importance of preparedness of the health care system for children based on systems approach, in the background of evolving knowledge of a pandemic and its outcome.

\section{Preparedness to COVID-19 Care}

The department is part of an academic institution based in coastal Karnataka, Southern India. We worked out the optimization of the services of the pediatric department to meet the following objectives:

1. To diagnose, isolate, and manage COVID-19 infected.

2. To reduce the risk of transmission to health care workers (HCW).

3. To rationalize the management of assets and resources.

4. To partner with the public health system.

5. To continue uninterrupted non-COVID services.

\section{Infrastructure Changes}

Fever clinic was established at the emergency department as the single-entry point to triage and segregate all children with fever, respiratory, and other COVID-19-compatible symptoms. We undertook infrastructure changes ( - Fig. 1) in the Children's Critical Care Complex as follows:

1. Separate entrance and exit for HCW and patients.

2. Donning and doffing areas for personal protective equipment (PPE).

3. Earmarked areas for stable and unstable COVID-19 suspect and COVID-19 positive, pediatric and neonatal (born to COVID-19-positive mother) beds.

4. Labor theater for COVID-19 suspect/ positive mothers.

The ICU had high-efficiency particulate air filtering system and negative pressure. We relocated the non-COVID-19 neonatal and pediatric beds for both regular and intensive care in other hospital areas. An inventory and stocking of medical products along with allocation of equipment like ventilators between the COVID-19 and non-COVID-19 areas were executed.

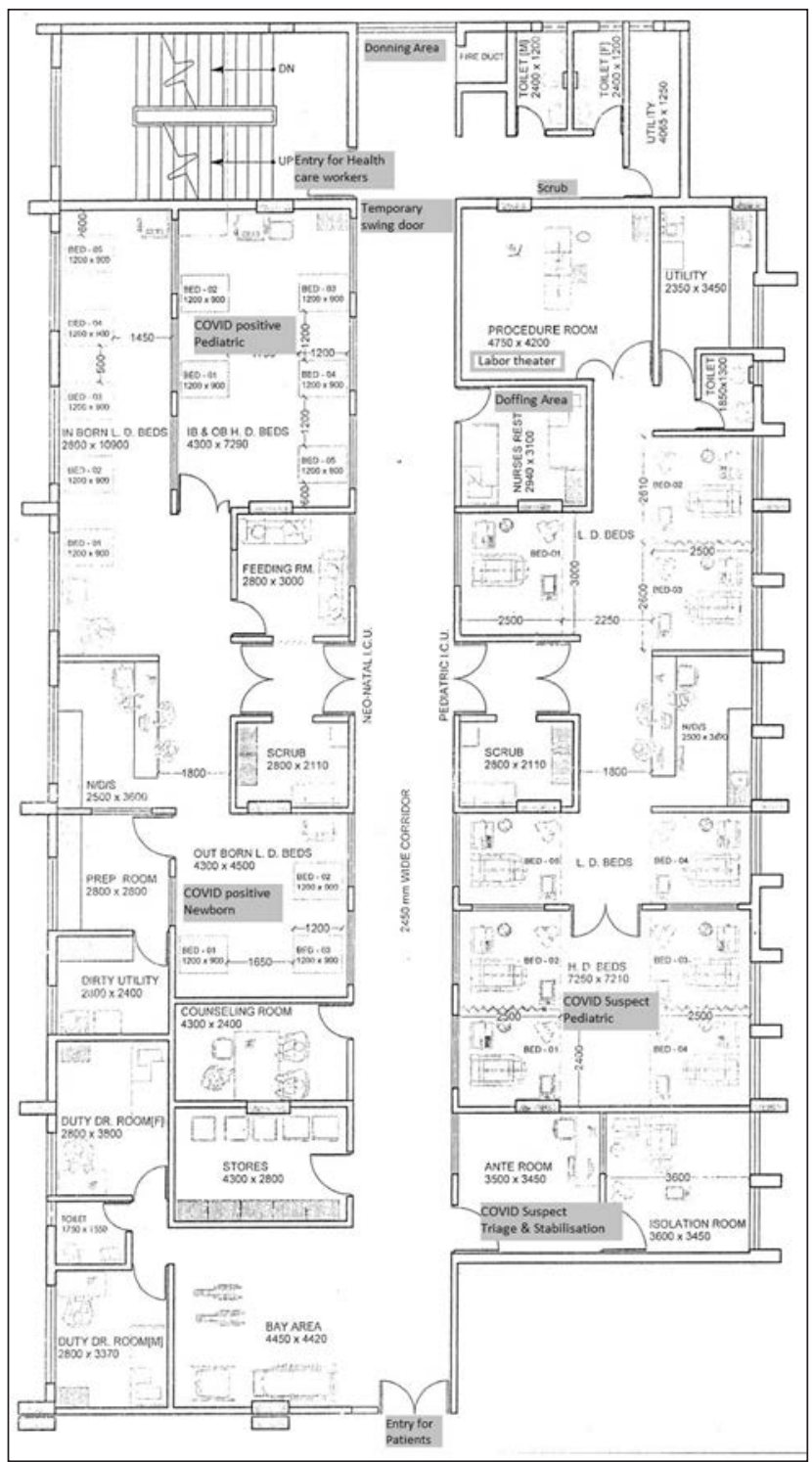

Fig. 1 Floor plan of Children's Critical Care Complex showing infrastructure changes for coronavirus disease 2019 admissions.

\section{Human Resources and Training}

A task force comprising of clinicians, intensivists, epidemiologist, microbiologist, biomedical engineering, and a member of the hospital administration board formulated the health system requirements and compiled the patient management protocols of individual departments..$^{14}$ It monitored the training of the HCW and support staff. Faculty and residents were reoriented to infrastructure changes, donning and doffing of PPE, changes to treatment protocols, especially intubation and ventilation strategies for minimizing aerosol generation.

Duty rosters for all HCW were reorganized into teams in a lean way, ensuring minimal staff exposure, no cross over between COVID-19 and non-COVID-19 areas, and adequate reserve staff. Ongoing changes to infrastructure and processes, clinical management guidelines, and government notifications were updated continuously and broadcasted using virtual platforms. The hospital board also focused on 
psychological support for the HCW and COVID-19-related biomedical waste disposal.

\section{Challenges in Health Care Delivery}

Optimization of services and safety of the HCW took precedence over cost and long-term repercussions on the department budgeting. We revisited the annual budgeting of the department. Issues with medical product companies, especially PPE, were the greatest challenge to the hospital. Telemedicine was expanded to provide continuity of treatment to chronic patients. Networking with community industries that could manufacture PPE coupled with existing buffer stock and rational use helped to tide over amidst the country-wide lockdown and underpreparedness very early on in the pandemic.

We worked in mutual cooperation with the district public health system. Our facility was part of the state contingency plan. We utilized the government facility for COVID-19 diagnostics until we established our own. The state also helped with contact tracing and surveillance. Google Spreadsheets working like an application were created to update ICU beds and ventilators in real-time across the district for adults and children.

\section{Outcome}

Our first admission of a critical case was on March 23, 2020 within 24 hours of the country going for a lockdown. He was a 10-month-old infant, presenting with SARI, hyperpyrexia, and seizures. He was designated as P06, the sixth case in the state, in the daily bulletin released on the COVID-19 information portal by the government. He was probably one of the first few infants with a severe disease in the country.

Over 6 months, between April and September, 22 children required in-patient care for an influenza like illness or SARI. We promptly recognized and managed MIS-C associated with COVID-19, in a 6-year-old girl presenting with myocarditis and shock in the second week of October.

A total of $33(6.5 \%)$ pregnant women tested positive for COVID-19; only 17 of the newborns were tested due to lack of consent of whom two (11.8\%) were positive. Two newborns had a severe transient tachypnea of newborn and had uneventful recovery. Their COVID-19 status was unknown. All babies were nursed at the mother's side.

The preparation also helped to manage childhood poisoning and trauma without cross-infection. Poisoning constituted $4.8 \%$ of PICU admissions in comparison to $2.1 \%$ during the corresponding period in 2019. Trauma constituted $4.2 \%$ of PICU admissions that were comparable to the corresponding period in 2019.

\section{Discussion}

In a health care system, the relationship between an operator, tasks, and contexts is dynamic. A pandemic like COVID-19 exposes the imbalance in exponential time; the health care facility requires rapid and drastic changes to infrastructure and functioning. ${ }^{15}$ However, we had difficulty assessing the extent to which these adaptations were needed very early in the pandemic, especially as the knowledge was scarce for the pediatric population. Children are generally a part of the family cluster. Dong et al from China, in one of the most extensive case series comprising of 2,135 children with COVID-19, reported that $\sim 43.9 \%$ had moderate-to-severe respiratory illness, and $0.6 \%$ had a critical illness with multiorgan dysfunction syndrome. ${ }^{6}$ The proportions of severe and critical cases were 10.6 and $7.3 \%$ among infants and children aged between 1 and 5 years. The authors reported only one mortality in the series.

Restructuring the health care system to deliver specialized COVID-19 care, the decline in routine services and regular office visits has led to losses amounting to hundreds of billion dollars for the hospitals. ${ }^{16}$ We adapted the systems approach and looked into the independent and interdependent modifiable factors. ${ }^{17}$ The key initiatives were to identify our objectives, bring out changes to the organizational processes, and integrate the same into the existing system. The strong commitment and reassurance to all employees' safety by the hospital board helped the department execute the infrastructure changes, reallocation of resources from its annual budgeting, roles and responsibilities of HCW, and handholding.

Our prior experience during Nipah virus outbreak in the neighboring state helped us quickly establish the fever clinic and protocols for segregation and triage. ${ }^{18}$ Our critical care unit with high-efficiency particulate air filtering system and negative pressure was best suited for management of COVID-19. ${ }^{19}$ Increase in indigenous manufacturing and innovations during the latter part of the pandemic ensured no imbalances in demand and supply. The management protocols relevant to our health care system were adopted..$^{14,19-21}$ This preparedness helped us manage COVID-19-positive children and neonates efficiently very early in the outbreak without collateral issues.

\section{Conclusion}

The preparedness of the health care system is crucial in a pandemic like COVID-19. This requires addressing all aspects of the system, namely, infrastructure, human resources, medical products, funding, leadership, and governance. Though the health system is mostly open, acting upon the modifiable factors using a systems approach gave better preparedness in a short time. Children also need prioritization as they are susceptible to the disease.

\section{Authors' Contributions \\ R.D.S., P.N., and S.S.R. were responsible for the planning and execution of the systems approach; A.M.J. coordi- nated with the district public health administration; S.H. supervised the changes in the health system approach. All authors have contributed equally to the conception and design of the manuscript. R.D.S. will be the guarantor.}

\section{Conflict of Interest}

None declared. 


\section{Acknowledgments}

The authors acknowledge the Board of Management of NITTE (deemed to be University), Dean, K.S. Hegde Medical Academy, and COVID Task Force, Justice K.S. Hegde Hospital for the kind support.

\section{References}

1 Qiu H, Wu J, Hong L, Luo Y, Song Q Chen D. Clinical and epidemiological features of 36 children with coronavirus disease 2019 (COVID-19) in Zhejiang, China: an observational cohort study. Lancet Infect Dis 2020;20(6):689-696

2 CDC COVID-19 Response Team. Coronavirus disease 2019 in children - United States, February 12-April 2, 2020. MMWR Morb Mortal Wkly Rep 2020;69(14):422-426

3 Shekerdemian LS, Mahmood NR, Wolfe KK, et al. International COVID-19 PICU Collaborative. Characteristics and outcomes of children with coronavirus disease 2019 (COVID-19) infection admitted to US and Canadian pediatric intensive care units. JAMA Pediatr 2020;174(9):868-873

4 Walker DM, Tolentino VR. COVID-19: the impact on pediatric emergency care. Pediatr Emerg Med Pract 2020;17(Suppl 6-1, Suppl 6-1):1-27

5 Hong H, Wang Y, Chung HT, Chen CJ. Clinical characteristics of novel coronavirus disease 2019 (COVID-19) in newborns, infants and children. Pediatr Neonatol 2020;61(2):131-132

6 Dong Y, Mo X, Hu Y, et al. Epidemiology of COVID-19 among children in China. Pediatrics 2020;145(6):e2020070210.1542/ peds.2020-0702

7 She J, Liu L, Liu W. COVID-19 epidemic: disease characteristics in children. J Med Virol 2020;92(7):747-754

8 Mehta P, McAuley DF, Brown M, Sanchez E, Tattersall RS, Manson JJ; HLH Across Speciality Collaboration, UK. COVID-19: consider cytokine storm syndromes and immunosuppression. Lancet 2020; 395(10229) :1033-1034

9 Verdoni L, Mazza A, Gervasoni A, et al. An outbreak of severe Kawasaki-like disease at the Italian epicentre of the SARS-CoV-2 epidemic: an observational cohort study. Lancet 2020;395(10239):1771-1778

10 Pathak EB, Salemi JL, Sobers N, Menard J, Hambleton IR. COVID-19 in children in the United States: intensive care admissions, estimated total infected, and projected numbers of severe pediatric cases in 2020. J Public Health Manag Pract 2020;26(4):325-333
11 Remy KE, Verhoef PA, Malone JR, et al. Caring for critically ill adults with coronavirus disease 2019 in a PICU: recommendations by dual trained intensivists. Pediatr Crit Care Med 2020;21(7):607-619

12 Fernandes ND, Cummings BM, Naber CE, et al. Adult COVID-19 patients cared for in a pediatric ICU embedded in a regional biothreat center: disease severity and outcomes. Health Secur 2020;10.1089/hs.2020.0225

13 Novel coronavirus disease - Situation update report 8 [Internet]. New Delhi: Ministry of Health and Family Welfare; 2020 Mar [cited 2020 April 24] p. 6. Report No.: 8. Available from: https://www.who.int/docs/default-source/wrindia/situation-report/india-situation-report-8bc9aca340f91408b9efbedb3917565fc.pdf?sfvrsn=5e0b8a43_2. Accessed May 26, 2021

14 NitteDU-KSHEMA handbook of COVID-19 protocols (version 3.1). Available at: http://www.kshema.nitte.edu.in/ img/pdf/covid-19-hb.pdf. Accessed June 10, 2021

15 Kim CS, Lynch JB, Cohen S, et al. One academic health system's early (and ongoing) experience responding to COVID-19: recommendations from the initial epicenter of the pandemic in the United States. Acad Med 2020;95(8):1146-1148

16 Blumenthal D, Fowler EJ, Abrams M, Collins SR. Covid-19 implications for the health care system. $N$ Engl J Med 2020;383(15):1483-1488

17 CheungR,RolandD,LachmanP.Reclaimingthesystemsapproach to paediatric safety. Arch Dis Child 2019;104(12):1130-1133

18 Ajith Kumar AK, Anoop Kumar AS. Deadly Nipah outbreak in Kerala: lessons learned for the future. Indian J Crit Care Med 2018;22(7):475-476

19 Ravikumar N, Nallasamy K, Bansal A, et al. Intensive Care Chapter of Indian Academy of Pediatrics. Novel coronavirus 2019 (2019-nCoV) infection: Part I - preparedness and management in the pediatric intensive care unit in resource-limited settings. Indian Pediatr 2020;57(4):324-334

20 Sundaram M, Ravikumar N, Bansal A, et al; Intensive Care Chapter of Indian Academy of Pediatrics. Novel coronavirus 2019 (2019-nCoV) infection: Part II - respiratory support in the pediatric intensive care unit in resource-limited Settings. Indian Pediatr 2020;57(4):335-342

21 Chawla D, Chirla D, Dalwai S, et al. Federation of Obstetric and Gynaecological Societies of India (FOGSI), National Neonatology Forum of India (NNF) and Indian Academy of Pediatrics (IAP). Perinatal-neonatal management of COVID-19 infection - guidelines of the FOGSI, NNF, and IAP. Indian Pediatr 2020;57(6):536-548 